\title{
Raison dans la folie et folie dans la raison dans l'œuvre de Medwall
}

Jean-Paul Debax

\section{(2) OpenEdition}

\section{Journals}

\section{Édition électronique}

URL : http://journals.openedition.org/shakespeare/1312

DOI : 10.4000/shakespeare.1312

ISSN : 2271-6424

Éditeur

Société Française Shakespeare

Édition imprimée

Date de publication : 1 novembre 1989

Pagination : 59-72

Référence électronique

Jean-Paul Debax, «Raison dans la folie et folie dans la raison dans l'œuvre de Medwall », Actes des congrès de la Société française Shakespeare [En ligne], 7| 1989, mis en ligne le 01 janvier 2007, consulté le 06 mai 2019. URL : http://journals.openedition.org/shakespeare/1312 ; DOI : 10.4000/ shakespeare.1312 


\title{
Société Française Shakespeare
}

\author{
Actes des Congrès \\ 1985 - 1986 - 1987
}

\section{LA FOLIE}

\section{SHAKESPEARE ET LES ARTS \\ LE TRAGIQUE}




\title{
RAISON DANS LA FOLIE ET FOLIE DANS LA RAISON DANS L'GEURE DE MEDWALL
}

\author{
Jean-Paul DEBAX
}

La société médiévale s'était peu à peu constitué un monde conceptuel à structure binaire où l'homme était tiraillé entre des principes et des forces opposés. Il y était constamment sollicité par un choix permanent entre des solutions opposées, des lieux antinomiques. L'espace qu'elle s'était créé était donc un espace contrasté, en noir et blanc, où les extrêmes se frôlent sans jamais se confondre, où les bons et les méchants sont séparés par un abîme moral, où le pouvoir de certains et la totale impuissance des autres font même douter que les uns et les autres appartiennent à la même humanité.

Même si timidement l'idée du Purgatoire est venue se glisser entre les extrêmes de félicité du Paradis et d'horreur de l'Enfer à partir du XIe siècle, même si l'artisanat et la bourgeoisie montante des villes prennent de plus en plus de place entre la classe dominante des nobles et des prélats et d'autre part le peuple des serviteurs et des paysans, ces modifications n'entament pas foncièrement le binarisme primitif : le Purgatoire n'est d'ailleurs conçu par l'église elle-même que comme une solution transitoire et provisoire avant le grand partage du Jugement Dernier, et il sera ensuite profondément ébranlé par les idées de la Réforme qui ne font que renforcer la bipartition originelle. La solution néo-platonicienne de la communion dans une unité amoureuse et paradisiaque n'est qu'un rêve dont les hommes ont toujours su qu'il était illusoire.

Quant aux divisions sociales, leur persistance prouve la profondeur de leur enracinement. La société sans classes n'est sans doute pas pour demain, et tant qu'il y aura des classes il y aura des haves et des havenots, des overdogs et des underdogs, des gens qui tiendront le manche et d'autres qui seront du côté de la cognée.

Ce manichéisme latent, bien que rejeté officiellement par l'Eglise Catholique, n'en est pas moins un puissant outil conceptuel, d'autant qu'il n'est pas sans garants dans l'Ecriture; l'histoire du monde, que les écrivains médiévaux se plaisaient à répéter inlassablement dans leurs traités théologiques et moraux, et qui est si magnifiquement représentée dans les Mystères des XIVe et XVe siècles, est fondée sur une altemance de périodes fastes et de temps d'épreuve, sur la succession 
d'événements agréables à Dieu et d'autres qui contrecarrent sa volonté (Chute des anges, péché d'Adam, immoralité des contemporains de Noë) et sur l'opposition de personnages bénéfiques et de figures démoniaques.

Alors que l'interprétation allégorique tend à susciter dans l'approche du réel la construction de niveaux superposés de correspondances entre les différents ordres de la création et ainsi à démultiplier la signification des événements ou des choses, de son côté la bipolarisation à tendance manichéiste a pour effet la constitution de séries verticales, où les objets et les concepts finissent par s'empiler, en se rassemblant autour de deux axes, marqués de signes de valeur contraires, où les spécificités des concepts se réduisent au profit d'un des deux signes de polarité : situation où la neutralité, l'indifférence ne sont pas pensables et se détruisent en s'assimilant à l'un des deux pôles opposés'. Allégorie et manichéisme additionnent ainsi leurs effets, aboutissant à une démultiplication indéfinie de la polysémie des termes, ce qui, conjugué avec une prolifération lexicale galopante, rend la tâche du critique particulièrement délicate.

C'est dans cette perspective de bipolarisation systématique que nous allons essayer d'étudier les deux termes de «folie» et de «raison» tels qu'ils apparaissent au XVe siècle, et ensuite d'envisager leur utilisation dramatique dans l'œuvre de Medwall en la comparant au thêâtre français des sotties.

A trois reprises dans sa moralité de NATURE Medwall fait allusion à l'opposition entre Raison et Folie. Dès le premier vers il prête à Raison un discours programmatique sur la destinée de l'homme :

Mais celui qui me fuit ou cause mon départ

Ill le regrettera des plus amèrement.

Il subira, c'est sûr, grande peine et tourment

D' avoir quitté Raison et poursuivi Folie, Et il s'en trouvera en dure pénurie.

A la fin de la première partie de la pièce, Genre Humain, plein de contrition après ses premiers errements, s'adresse à Raison :

$O$ divine Raison, $j$ ' ai un plus grand besoin

De votre bon secours que jamais je n' ai eu.

Aidez-moi aujourd' hui, ne vous quitterai plus. 
Depuis que je vous ai trahie

Je fus la proie de cent folies.

Plus loin encore, Sensualité lui fait reproche de cette conversion et lui demande dans un oxymore particulièrement percutant comment il a pu suivre les conseils «fous» (foolish counsel) de la Raison ${ }^{2}$.

Il n'est pas question d'entreprendre ici une histoire des termes «raison» et «folie», mais de faire un bref rappel pour situer leur acception au moment où Medwall s'en emparait.

Une des premières manifestations littéraires de l'opposition binaire entre un principe de sagesse et son contraire à l'intérieur de l'âme humaine se trouve dans un texte homilétique en Moyen-Anglais, des environs de 1200, SAWLES WARDE (La Garde de l'âme), en forme de commentaire allégorique sur le texte de Matthieu : «Si le maûtre de maison savait à quelle heure de la nuit le voleur doit venir il veillerait et ne laisserait pas percer sa maison» ${ }^{3}$. L'esprit de l'homme y est figuré comme une maison dont le maître est Wit (terme que nous pouvons traduire par sagesse, esprit, raison), et dont l'imprévoyance maîtresse est Will (l'impulsion, le désir, la fantaisie, en d'autres termes «la folle du logis»). L'histoire se déroule comme une petite psychomachie bourgeoise et domestique pleine de vie : tout autour de la maison les vices sont à l'affût ; ils convoitent le trésor caché dans la maison : l'âme humaine. Pour stimuler la vigilance des défenseurs, Prudence dépêche Peur, l'envoyé de la Mort, et ensuite Amour-de-la-Vie-Eternelle qui, chacun par ses arguments propres, arrivent à réconcilier Wit et Will, c'est-à-dire Raison et Folie, et la force retrouvée par leur union leur permettra de repousser les assauts menaçants des vices.

Cette même opposition est illustrée au XVe siècle par Lydgate dans l'Assemblée des dieux dont le sous-titre est assez éloquent : «L'accord de raison et de sensualité dans la peur de la mort». La scène, comme dans SAWLES WARDE est le microcosme. Les rôles sont distribués selon un schéma qui rappelle celui de l'allégorie moyen-anglaise : les vices, les vertus, les cinq sens se retrouvent dans les mêmes emplois ; mais deux nouveaux acteurs apparaissent chez Lydgate portant les noms de «Raison» et «Sensualité». L'important n'est pas au premier chef les valeurs que nous pourrions attacher à ces deux termes en vertu d'une filiation étymologique. Il se peut que le terme de raison rappelle l'âme raisonnableou «logistikon» de Platon, ou la «raison» de l'amour courtois : (le «fine amor») où elle s'oppose justement à «delit», le désir charnel, l'œuvre de chair, l'abandon aux passions sensuelles, qui n'est autre donc que la sensualité, cet abandon que, dans son TRISTAN, Thomas d'Angleterre nomme justement le «voleir» ou «volente», ce qui évoque pour nous le Will déjà rencontré dans SAWLES WARDE ${ }^{4}$. Mais une incertitude demeure, due à la polysémie découlant d'une possible allégorisation : Raison, comme Sensualité, font-elles allusion à l'ordre du monde, à celui de l'amour courtois, ou à celui de l'ordre théologique qui nous parle des rapports de l'homme avec Dieu ? Ce qui est 
important c'est de saisir la relation de ces deux mots, comme représentant des concepts contradictoires, et donc mutuellement exclusifs qui vont se définir l'un l'autre dans un contexte donné.

Le terme de folie a envahi brusquement l'espace social ainsi que l'imaginaire des hommes avec le début de la Renaissance, nous dit Michel Foucault ${ }^{5}$. Les mêmes rites et les mêmes structures d'exclusion qui avaient frappé les lépreux tout au long du Moyen-Age se reportent alors sur ceux qu'on appelle les «fous», et qui semblent se mettre à exister par l'exclusion même dont ils sont l'objet. La léproserie cède la place à l'asile, le ladre rejeté est remplacé par l'aliéné itinérant ${ }^{6}$. Cette vision horrible de la mort incarnée par les danses macabres du moyen-âge finissant est maintenant détrônée par cet autre symbole du «grand pàssage», inquiétant, mais plus allusif et plus ambigu aussi, la Nef de Fols. Toute l'inquiétude des hommes se reporte donc sur les fous et sur la folie ; et bien qu'il y ait une certaine ambiguïté de la notion de folie, ce concept vient cependant se loger sur le pôle négatif de l'opposition binaire déjà mentionnée, où elle voisine aisément avec Will, dont nous avons déjà vu les connotations péjoratives, voisinage qui durera jusqu'à la fin de la période élisabéthaine comme l'illustrent ces quelques vers de l'Urania, de John Taylor.

When Wisedome must give Follie cap and knee
When hare-brained Will o' re Wit doth rule and raigne

When they have Reasons Court thus underminde, It is a signe that Understanding's blinde.

Quelques années après que Sebastian Brant eut décrit la vie et la mort des hommes en termes d'une étrange navigation, symbole de l'enfermement et du destin inexorable de l'humanité, Erasme consacre le règne de cette nouvelle déesse des hommes en écrivant son Eloge dont la caractéristique fondamentale est l'ironie et l'ambiguïté, à l'image même du narrateur de l'œuvre : puisque l'éloge écrit par la folie elle-même ne peut être que folie. Que devons-nous penser alors des dénonciations contenues dans ce volume, en particulier celles portant sur l'existence même du monde : par deux fois Erasme fait allusion aux ombres de la caverne platonicienne : «Trouvez-vous une différence», écrit-il, «entre ceux qui, dans la caverne de Platon regardent les ombres et les images des objets, ne désirant pous rien et $s^{\prime} y$ plaisant à merveille, et le sage qui est sorti de la caverne et qui voit les choses comme elles sont ? "7. Comme nous le savons, le sage qui vient révéler à ses anciens compagnons leurs illusions, est tourné en dérision par eux et, en fin de compte, chassé. Et qui nous garantit d'ailleurs que le «sage» était arrivé à une vraie connaissance, et n'était pas victime, une fois sorti de la caverne, d'une nouvelle illusion ? Comme le sage, Folie, narratrice de l'Eloge de la Folie, est un point de vue extérieur, une instance qui devrait garantir la vérité de ce qui est dit, mais justement, si elle est folie elle ne le peut pas. 
La substitution de «folie» à mal, à péché, à Monde et à Sensualité» dans la grande alternative qui préside aux choix humains selon l'optique chrétienne, ne témoigne pas d'un simple changement lexical, ou même sémantique. Avec l'introduction de Folie dans le système, un point extrême est atteint où les concepts basculent, où la possibilité même de l'opposition, s'abolit. La surface même du langage se met à représenter le chaos et l'incohérence du monde. Non que cette surface soit l'image ou l'expression d'un doute généralisé ou d'un nouvel athéisme : nous savons bien que c'était tout le contraire dans le cas d'Erasme dont la foi était sereine et profonde; mais l'introduction de la folie crée un effet parallèle mais inverse de celui de l'interprétation allégorique ; cette dernière accumule les sens dans une perspective infinie et cumulative. La folie les empile pour qu'ils se détruisent successivement de façon dialectique, et en fin de compte les dérobe à la vue du lecteur et lui restitue sa liberté, liberté dont la manifestation la plus éclatante est inscrite dans l'œuvre de Rabelais ${ }^{8}$.

«Dans cette extravagance le théâtre développe sa vérité, qui est d'être illusion. Ce qui est, au sens strict, la folie» écrit Michel Foucault en conclusion de sa description de la généralisation du thème de la folie à la fin du moyen-âge. Au thêatre le personnage est toujours illusion, mais illusion d'une personne. Le fou aussi, qui ne juge pas sainement des choses et se trompe sur sa propre nature est illusion. Quand le fol accède au théâtre il y est comme illusion d'une illusion, le personnage le plus thêatral qui soit et l'image même de l'illusion théâtrale.

Le XIVe siècle invente un théâtre dont tous les personnages sont des fols : c'est la sottie. Si, dans le théâtre français, la sottie existe comme un genre à part, elle partage pourtant bien des traits avec les genres voisins, la farce et la moralité : tous trois ont en commun des scènes de comique physique, des bastonades, des quiproquos. La sottie partage avec la moralité les personnages allégoriques ou typiques, et un genre d'intrigue très particulier, non motivée et qui se résoud à la réalisation d'une plaisanterie malveillante jouée par certains personnages à l'encontre et au détriment d'un autre. Si la sottie n'existe pas comme genre dans le théâtre anglais, l'esprit de la sottie en est-il totalement absent ? Je propose au contraire de voir dans maint personnage de ce théâtre des fols naturels ou artificiels, ainsi que des similarités de structure.

Voyons ce qu'il en est dans l'œuvre de Medwall.

On se souvient que dans WISDOM, l'une des plus anciennes «moralités» anglaises, Lucifer révèle, bientôt après son apparition, un habit de «galant orgueilleux» sous son manteau de diable. C'est dans ce costume «révélé» qu'il va agir dans le monde et essayer d'acculer l'esprit de l'homme au désespoir'. Ce terme de «galant» n'est pas inconnu dans les interludes anglais. Youth, dans l'interlude de 
YOUTH, traite de «galant» les vices qui mènent en sa compagnie une vie de débauche ${ }^{10}$. Ce terme est aussi utilisé quatre fois dans l'œuvre de Medwall, deux fois dans NATURE et deux fois dans FULGENS AND LUCRES ${ }^{11}$. Or nous découvrons que dans la sottie «galant» n'est qu'un des nombreux synonymes de «sot, fol, bru, compagnon, suppot, esbahy ou gens nouveaulx»...12. Comme dans WISDOM, dans plusieurs sotties les fols révèlent leur costume de fol caché sous un autre costume. On rapprochera cette caractérisation de la nature du personnage par le costume qu'il porte, de l'importance donnée à la mode vestimentaire chez les «fols» de l'œuvre de Medwall. Genre Humain et Orgueil de NATURE sont tout accaparés par ce problème de costume, moyen et symbole de leur déchéance. De même dans FULGENS AND LUCRES c'est Cornelius, le prétendant vicieux qui n'obtient pas la main de Lucrèce, qui se signale par le luxe de ses atours, et il est ironiquement celui des deux qui est l'esclave de la mode, alors qu'il se pose comme le défenseur de l'ancienne noblesse et des vertus de l'hérédité ${ }^{13}$. Comme l'habit de fol dans la sottie, leur luxueux costume est à la fois déguisement et dévoilement. Ni Genre Humain ni Orgueil ne sait que l'autre est un fol. Dans FULGENS AND LUCRES, A et B jouent le jeu avec Cornelius, tout en mettant à jour ses travers et sa folie dans une relation ambiguě et constamment ludique. Dans tous les cas la nature de «fols» de ces personnages est claire pour le public : le fol est toujours victime en même temps que trompeur.

La sottie est un jeu de la séduction ; c'est aussi un jeu du pouvoir. Dans NATURE, comme dans bon nombre de pièces en forme de moralité, l'homme est toujours aussi le souverain. Comme celle de MAGNIFICENCE ou de ANE SATIRE, l'action de NATURE est aussi bien morale que politique, si nous faisons là une distinction qui n'avait pas cours en cette fin du moyen-âge. Le rite d'intronisation auquel nous assistons dans les premières scènes nous le confirme. L'imitation, qui est en même temps une parodie, des liturgies civiles et religieuses (processions, funérailles, baptêmes, adoubements) est un jeu de scène classique dans les sotties ${ }^{14}$. Les textes que nous possédons, même s'ils n'en portent pas la trace, devaient être accompagnés de force pitreries et gesticulations, le plus souvent comiques sinon toujours très convenables. Ce serait à mon avis un contre-sens que de jouer sérieusement ce cérémonial initial et initiatique dont l'inversion est manifestée par le renversement de sens des mots wise et goodly ${ }^{15}$. Le Monde conclut la séance d'habillage par des termes sur lesquels, bien évidemment, le public ne se méprend pas. S'adressant à ce Genre Humain, il lui dit :

Et tu es le plus beau qui n'ait jamais été, Tu vaux mille fois plus qu' avant de t' habiller. (I $N, 468-9$ )

FULGENS AND LUCRES également, est une pièce sur le pouvoir. On a pu l'interpréter comme le mariage allégorique de l'Angleterre avec les hommes 
nouveaux de l'entourage de Henry Tudor, de préférence aux vieux barons féodaux concurrents du pouvoir royal. FULGENS AND LUCRES tient du débat dramatisé et de la «sottie-jugement», puisqu'un juge est choisi d'un commun accord par les deux parties pour trancher la controverse : pour la commodité de la pièce ou pour appuyer son sens allégorique, c'est à Lucrèce elle-même qu'est conféré cette responsabilité.

Dans FULGENS AND LUCRES comme dans NATURE la comédie du pouvoir est mêlée au jeu érotique ; autre manifestation du pouvoir, Genre Humain, après avoir satisfait ses goûts pour le luxe du costume, cherche par deux fois la compagnie de demoiselles accueillantes. Dans FULGENS AND LUCRES, outre le fait que la structure d'ensemble de la pièce est une allégorie romantique, les symboles érotiques se mêlent à ceux du pouvoir. Les connotations érotiques sont confirmées par la présence de l'intrigue dite «secondaire», constituée par la cour que font les serviteurs A et B à Joan la servante de Lucrèce, intrigue qui fait écho à l'intrigue «principale» concernant Lucrèce et ses deux prétendants. L'érotisme se manifeste à l'intérieur de cette seconde intrigue dans le tournoi parodique que livrent A et B et qui s'accorde bien avec leur «basse» condition de serviteurs. Cette compétition porte le nom pittoresque de Fart-pryke-in-cule, et devait constituer un spectacle fort réjouissant, puisqu'il s'agissait d'une lutte où les antagonistes s'affrontaient accroupis, avec, semble-t-il, un bâton passé sous les genoux auquel les mains étaient attachées : pugilat évocateur d'un combat de coqs, cette allusion renforçant encore la charge érotique du jeu ${ }^{16}$.

Autre exemple, l'histoire rapportée par B à Lucrèce d'une rencontre que cette dernière a eue avec Cornelius et au cours de laquelle Cornelius avait chassé un oiseau indiscret en lui lançant la boule à parfum de Lucrèce. Les connotations érotiques sont d'abord fournies par le vocabulaire : l'arbre sur lequel s'est posé l'oiseau est un frêne = ash ; or, au XVe siècle arse se prononce «ash», et de plus, cet arbre est creux, et donc percé d'un trou. Remarquons aussi que Cornelius indique que la boule en question y a atterri comme un épieu (pole). On précisera enfin que l'oiseau en question est un coucou... Mais aussi Cornelius, sa boule à la main, ressemble irrésistiblement au fol tenant sa vessie, symbole sexuel aussi, tout autant que de folie. Cette boule à parfum ne fait-elle pas penser aussi à la pierre de folie qu'on voit les fols serrer dans leurs mains dans certaines sculptures du temps?

Le «badin» est une variété de fol naturel qui peut se définir par son innocence ${ }^{17}$. Deux détails se rapportant au personnage de Glouton dans NATURE nous font penser que c'est un personnage de badin. Il se dispute d'abord avec Amour Mondain comme le font deux clowns sur la piste d'un cirque, à propos d'un tabouret : «je veuxm' asseoir» - '«non tune vas past' asseoir»-«qu' est-ce que c'est que celui-ld qui veut toujours commander ?»...18.

Comme son nom l'indique il ne pense qu'à manger. Mais ce qui nous intéressera davantage c'est l'arrivée de Glouton, un fromage et une bouteille à la main : symboles de sa gloutonnerie, certes, mais marques de son statut populaire aussi, puisque le fromage est la nourriture du pauvre et symbole de la vieillesse aussi 
puisque l'ivrognerie est plutôt, avec l'Avarice, un péché de vieillard ${ }^{19}$. Mais surtout ce fromage rond porté d'une main et la bouteille tenue droite dans l'autre font de Glouton un roi tenant un orbe et un sceptre parodiques, insignes d'une majesté carnavalesque : vision fugitive certes, dans la pièce, mais reflet probable de la pratique des fous de cour, tel ce Martin, surnommé le Bailli, fou de Henri II de France, qui portait une copie en laiton du collier de l'ordre de St Michel ${ }^{20}$.

Contrairement à la sagesse dont devrait faire preuve un roi, Glouton s'embrouille dans ses salutations, plaisanterie qui est répétée mot pour mot par le serviteur A de FULGENS AND LUCRES : «mon maitre me recommande à vous» - "Quoi vous a moi ?»... autre badin, tout au moins temporairement ; lequel A est tellement sot qu'il en oublie même son propre nom ainsi que celui de son maitre ${ }^{21}$.

L'action du fol n'est pas motivée ; elle est dénuée de logique. Par cela elle s'oppose dans son principe même à la notion de raison. Les sots des Trois galants et Monde et de Deux galants et société sont de cette espèce, eux qui mettent en pratique l'adage que «chacun a par soy sa régente». Leur maître c'est leur volonté incontrôlée, Will que nous avons déjà rencontré dans SAWLES WARDE, et qui jouera souvent le rôle du Vice sous les noms de Freewill ou de Ill-Will. Envie dans NATURE déclare ouvertement appartenir à cette espèce de vauriens : après qu'il a joué ce tour pendableà son camarade Orgueil de lui faire croire qu'il est arrivé après la bataille et qu'il est renvoyé de la bande de retainers armés au service du seigneur Genre Humain, Sensualité lui demande :

Mais quelle folie inutile?

Dis-moi, quel est donc ton mobile?

il lui répond :

En vérité je n' en ai pas,

C'est simplement ma fantaisie

(II $N$, 919-22).

La satire sociale est un locus communis de la sottie ${ }^{22}$. Le sot y fait souvent figure de personnage populaire critiquant la classe dominante. Par définition, le peuple est un exclu qui peut observer de loin les gesticulations du pouvoir, comme le sage de Platon voyait les prisonniers et les ombres dans la caverne : par ce biais s'introduit le thème de la sagesse du fou ${ }^{23}$. Dans NATURE Genre Humain et Orgueil appartiennent à la noblesse : ils sont tour à tour trompeur et trompé. Raison est tournée temporairement en ridicule, mais dans le cadre de la moralité c'est évidemment elle qui a le dernier mot. Dans FULGENS AND LUCRES, Cornelius est aussi un puissant et paraît comme un vrai fol qui finira trompé. C'est lorsque Lucrèce sort de son rôle de Dame courtisée pour jouer avec $A$ et $B$, qu'elle devient sage dans le domaine du non-pouvoir. Mais c'est surtout A et $B$ qui font les aller et retour les plus 
fréquents entre folie et raison. Ils opposent la sagesse populaire à la folie des grands, l'honnêteté populaire à la fourberie des grands :

B «Le monde, de nos jours force-t-il à mentir «Et à prétendre que le corbeau est tout blanc?

A *Oui, je crois bien qu' on doit paraûtre complaisant ; «Et il lui faut flatter et entrer dans la ronde *Celui qui veut compter parmi les gens du monde.

«A la cour de tels gens seront très appréciés.

B «Oui, mais dans la paroisse où j' ai mon logement «Flatterie est tenue pour le pire péché. «Les menteurs sont honnis particulièrement «Dès qu'on voit ce péché dans leur comportement»

(I $F \& L, 163-72)^{24}$.

Il n'est pas question pour le fol populaire d'affirmer systématiquement la supériorité du peuple, surtout si, comme c'est le cas ici, la pièce est jouée devant des artistocrates. Le point de vue populaire n'est présenté que par l'un des deux fols tandis que l'autre prend le contrepied de ses affirmations.

Une manifestation de la sagesse populaire est, en dehors de tout jugement de valeur, d'accepter le verdict des faits, comme le suggère $B$ à propos de Cornelius qui n'a pas obtenu la main de Lucrèce :

Maintenant que le mal est fait

Il doit bien s'en accomoder

Et déclarer qu'il est content

(II $F \& L, 815-7)$.

Au contraire en apprenant sa déconfiture Cornelius en sera fou de rage (stark mad).

Une solution extrême est pour les fols de s'affirmer complètement extérieur aux valeurs de la société, contestation radicale s'il en est, et ainsi de poser au vrai cynique ${ }^{25}$. A termine FULGENS AND LUCRES sur ces paroles dignes de Machiavel :

La vertu, qu' est-ce que c'est donc?

Capasse l' imagination!

Qu'est-ce que ça peut vouloir dire?»

(II, $F \& L, 842-4)^{26}$. 
Au-delà de leur condition de fols comiques, acrobates, chanteurs, manipulateurs de leurs camarades et du public, A et B accèdent à la dignité de «morosophes» ou de «fols-sages» : et «dignité» n'est pas lancé au hasard, puisque depuis la révélation évangélique le vrai sage n'est pas celui qui suit la sapientia : la véritable raison est ce qui aux yeux du monde fait figure de déraison et de folie... En ce sens le premier fol est le Christ lui-même ${ }^{2}$.

Comme Triboulet, le fol de cour de Louis XII, comme Marcolphe fou de Salomon, nos compères A et $\mathrm{B}$ en arrivent à être «le seigneur souverain de tous ceux dont il se moque». S'ils ne se moquent pas ouvertement des deux seigneurs Gaius et Cornelius, ils accaparent l'intrigue de ces demiers, y entrant par effraction pour la faire leur, et lui imposer un rythme qui est le leur propre. Ils les possèdent dramatiquement ${ }^{28}$. Comme le Triboulet de Rabelais qui permet la relance de l'enquête qui conduira en fin de compteà la Dive Bouteille, et de qui vient la réponse définitive au cinquième livre, et qui, parce qu'il donne son sensà l'œuvre, s'identifie à l'auteur-démiurge lui-même, de même nos serviteurs, présentateurs, interprètes, manipulateurs, A et B sont l'image du dramaturge créateur d'un monde qui tient entre ses mains toute folie et toute raison ${ }^{29}$.

L'image de cette inclusion mutuelle de la folie et de la raison nous est enfin fournie par la structure des deux pièces. Elles sont en effet construites sur un modèle unique. Dans NATURE le dialogue initial de Raison et de Sensualité donne naissance à un autre niveau de jeu, celui du Monde intrônisant Genre Humain, dont la pièce va rapporter les aventures, qui, elles-mêmes, englobent d'autres scènes ou récits enchassés plus ou moins reliés thématiquement à cette intrigue "principale». De même, dans FULGENS AND LUCRES, A et B semblent être là pour introduire une intrigue. En fait eux aussi se trouvent pris comme acteurs d'une action propre ; tout cela englobant encore des scènes enchassées, des «shows», des «récits». Ces structures, qui ont été diversement décrites comme «en sandwich», «en noyau et écorce» et «en pelure d'oignon», proposent une alternance de niveaux de jeu qui peut être lue comme une alternance entre raison et folie. Il semble à première vue que NATURE introduise la folie dans un cadre raisonnable, celui de la moralité, alors que FULGENSAND LUCRES introduit la raison du débat sérieux dans la folie du cadre proposé par les «tricksters» A et B. Mais ce qui est plus significatif que cet enveloppement de la pièce dans un emballage, une "présentation», en forme d'écorce ou de peau d'oignon, c'est la présence même de ruptures successives qui modifie sans cesse le regard qu'on peut porter sur la pièce, et qui interdit donc une définition constante et confortable du destinataire, puisque l'acteur. d'un instant devient brusquement narrateur ou spectateur l'instant d'après. Cette figure narrative, à la suite de Genette, je l'appellerai «métalepse». Et, écrit Genette : «Le plus troublant de la métalepse est bien dans cette hypothèse inacceptable et insistante, que l'extra-diégétique est peut-être déjà diégétique, et que le narrateur et les narrataires, c'est-à-dire, vous et moi, appartenons peut-être encore à quelque récit» ${ }^{30} . \mathrm{La}$ 
métalepse, structure basée sur l'éclatement, imposè la recherche d'un principe unificateur. La métalepse est comme une injonction adressée au spectateur qui lui impose de prendre un rôle actif dans le commerce théâtral, qui l'inclut de force dans les gesticulations du monde sublunaire, qui lui prouve qu'il n'est point de sage qui puisse s'absenter de la caverne de Platon, que Sirius n'existe pas, et qu'il est luimême embarqué sur la Nef des Fols. 


\section{NOTES}

1 Cette bipolarisation ne signifie pas l'abandon de toute structure trinaire, comme la division de la société en trois «états», la Sainte Trinité, et la trinité inversée des puissances maléfiques : le diable, la chair et le monde ; au théâtre, un groupe fréquent de trois vices sous l'autorité d'un leader, ou capitaine (les sots des Sotties reconnaissent aussi lạ nécessité d'être trois : Heather Arden, Fools' Plays, Cambridge, 1980, p. 37), et, dans le microcosme, les trois puissances de l'âme - esprit, volonté, entendement peut-être héritées du platonisme et reprises par St Augustin.

2 II NATURE, 70.

Ailleurs Genre Humain rapporte que Raison lui a interdit d'obéir à sa «fantaisie» (fantasye), ou d'accorder quelque crédit à son propre jugement (Wit), I N., 974-7.

3 Mat. $24 ; 43$.

Sawles Warde est une adaptation de quelques chapitres du De Anima de Hugues de St Victor ; texte dans J. Hall, Selections from Early Middle-English, 1130-1250, Oxford, 1920, 117-28.

Comme texte antérieur à Sawles Warde, on peut citer l'Eneas de 1160, où apparaît un débat entre Raison et Folie. «Lavine, fiancée à Turnus aime Enée qui est l'ennemi de son lignage. Ce déchirement intérieur se traduit par l' affrontement entre Lavine folle et Lavine sage», J. Ch. Payen, «Genèse et finalité de la pensée allégorique au Moyenâge», Revue de métophysique et de morale, 1973, 466-79, p. 471. On rapprochera ce dédoublement des deux Iseult qu'aime Tristan : Iseult aux blonds cheveux et Iseult aux blanches mains.

On peut mentionner également le dialogue fragmentaire :The Conflict of Wit and Will, circ. 1400, ed. Bruce Dickins, The School of English Language in the University of Leeds, 1938.

4 Voir le commentaire de J. Frappier, «Sur le mot "Raison" dans le Tristan de Thomas d'Angleterre», Linguistic and Literary Studies in Honor of Helmut Hatzfeld, ed. A.S. Crisfulli, Washington, 1964, 163-76, p. 174, n. 46. Chez St Bernard Raison prend le sens de «faculté supérieure de l'âme». Frappier montre bien la similitude entre l'emploi théologique de raison et celui qui en est fait dans la littérature courtoise où Amour et Raison se confondent.

5 Histoire de la folie à l' âge classique, 1961, 13-53.

6 Tristan revenant au château de Marc se déguise, pour signifier son exclusion, en «fou» ou en «lépreux» (selon les versions). Voir J. Batany, Approches du "Roman de la rose", Paris, 1973, 97-101.

7 Erasme, Eloge de la folie, Traduc. P. de Nolhac, 1964, XLV. 
8 Voir Lucien Febvre, Problème de l' incroyance au XVIe siècle, 1942, Me partie, Ch. III sur Rabelais et Erasme.

9 «And aftyr be songe entreth LUCYFER in a dewyllys aray wythowt and within as a prowde galonte,.... (didascalie après le v. 324)

«Her LUCYFER dewoydyth and cummyth in ageyn as a goodly galont» (didascalie après le v. 380).

De même dans Le Prince des sots, le Prince entre habille d'une longue robe, mais «descubs est habillé en sot» (cité Arden, 1980, p. 49)

H. Arden suggère, à juste titre, que ces déshabillages forcés ou volontaires révèlent la dualité des personnages: «A sot, as well as a sottie, may have two levels: the "official" character, indicated by the fool's costume that he wears on entering, and the "real" one hidden beneath the other ». (Ibid.p. 49).

10 Youth «Aback, gallants, and look unto me, *And take me for your special» Youth, 580-1 in E. Schell et J.D. Schuchter, English Morality Plays and Moral Interludes, New York, etc, 1969.

11 I NATURE, $1059 \mathrm{ET} 1078$, I F \& L 54 et 586.

12 Voir Arden, 1980, p. 37.

13 If $F \& L, 717-50$ et II $F \& L, 451-535$.

14 Citons La sottie nouvelle des premiers gardonnez, 1480, Les vigilles de Triboullet, 1470 ou Les sots nouveaulx, 1513.

15 IN, 442, 446 et 467.

16 Pour la description du «Farte-pryke-in-cule» on consultera P. Meredith et Meg Twycross, «Fart Pryke in Cule and Cock Fighting», Medieval English Theatre, 6.1, $1984,30-9$.

17 II N, 786-8

J.-C. Aubailly donne cette définition du badin : «le simple d'esprit "par nature" ou badin (défini face à la société par un rapport d' exclusion-inférioritê)», Le monologue, le dialogue et la sottie, 1973, p. 354.

18 IIN, 516-25.

19 Ibid. didascalie après 757. «Il est pourtant des gens, surtout le vieil âge, plus amis de la bouteille que de la femme qui trouvent leur bonheur suprême en beuveries», Erasme, Eloge, XVIII.

20 Cité Lever, Le sceptre et la marotte, Paris, 1983, p. 197.

21 II N, 792-6 et II $F \& L, 316-53$.

Cet oubli du nom n'est pas exceptionnel dans les interludes : c'est le cas de Falset, Ane Satyre, 858 ; de Ambidexter, Cambises, 148 ;Respublica, 370-415 et Enough, 473-89.

22 Voir Arden, 1980, pp. 166-7 et Ch. 5.

23 On appréciera l'ironie qui vient de l'opposition des classes sociales :

«Well, as for gentilman, it is full kynde *To have theyr plesyrs that may well payex

I $F \& L, 751-2$.

et $B$ déclare que le but de la pièce est:

*...that suche as be gentilment of name

"may be somwhat movyd

«By this example for to eschew

"The way of vyce and favour vertue....

II $F \& L 891-4$ 
24 «Les «sages» ont deux langues... l'une pour dire la vérité, $l$ ' autre pour dire ce qui est opportun. Ils savent "changer le noir en blanc"»... Eloge XXXVI ; Flatterie est scur de Philautie, Eloge, XLIV.

25 «Mother Folly became the archetype of XVth. century cynicism», B. Swain, Fools and Folly during the Middle Ages and the Renaissance, N. York, 1932, p. 91 ; cité Arden, 1980 , p. 160 , n. 1.

26 On rapprochera ceci de :

«How be it, the matter touchith me never a dell, «For I am nether of vertue excellent «Nor yet of gentyl blode».

I $F \& L, 140-2$

27 Ce retournement des polarités est bien mis en évidence par Joël Lefebvre, Les fols et la folie, Paris, 1968, p. 20 :

«L'opposition folie-sagesse de l'Ancien Testament subit un renversement dans les Evangiles, où la «stultitia» reçoit une acception positive dans de nombreux passages... La véritable sagesse, ce n' est plus la «sapientia» : l'ensemble des règles définies par l'Ancien Testament".

Cette inversion et illustrée par les ascètes médiévaux qui abandonnent par «folie» leur statut social et les biens de ce monde. Le cas extrême est représenté par les salö̈ orientaux qui assument un statut de «fou», comme le très populaire St Alexis qui, issu d'une grande famille romaine, part comme ermite en orient, et revient ensuite à Rome incognito, et couche sous l'escalier, comme un clochard, dans la maison de son propre père. (Cf. J. Batany, 1973, p. 98).

Même aujourd'hui certains intégristes musulmans ne s'appellent-ils pas *fous de Dieu»?

28 Un exemple révélateur est la façon dont A et $B$ bernent «ludiquement» Cornelius, en lui faisant croire que la raclée qu'ils ont reçue de Joan leur a été administrée par les serviteurs de Gaius (I $F \& L, 1245-85$ ) bien que Comelius révèle ensuite (pour eux et pour le public) qu'il n'a pas «marché» :

«Well then, ye lye both two !» 1286.

29 Cf. Lever, 1983, p. 182.

30 G. Genette, Figures III, 1972, p. 245. 